regarding the need for escalation and which children we have greater concern for, leading to early identification of these patients.

Lessons learnt Implementation requires buy-in and consensus of the whole team. Next time I would provide more regular feedback of the success of change to ensure continued motivation of users and highlight areas for improvement.

Message for others An extremely simple intervention can help to improve efficiency and have a positive effect on patient care safety by prompting key points in patient management, especially in a busy environment.

\section{G548(P) SPECIAL REQUIREMENTS IN BLOOD TRANSFUSION - AN AUDIT OF REQUESTING ERRORS}

II Morgan, ${ }^{2} \mathrm{E}$ Harrison, ${ }^{3} \mathrm{~F}$ Nicholson. 'Medicine, Royal Hospital for Sick Children, Glasgow, UK; ${ }^{2}$ Blood Bank, Royal Hospital for Sick Children, Glasgow, UK; ${ }^{3}$ Haematology, Royal Hospital for Sick Children, Glasgow, UK

\subsection{6/archdischild-2015-308599.498}

Context This Audit was conducted in a tertiary haematology/ oncology unit to identify errors in red cell transfusion requests by unit nursing and medical staff.

Problem When requesting blood it is important to consider whether the patient has any special transfusion requirements, most common being the need for CMV screened or irradiated blood products. It is important to request these for patients who require them and significant harm can be caused to patients if they don't receive what they need (Figure 1). It is equally key to avoid wasting these resources; Irradiation reduces shelf life of blood, and over-requesting CMV negative blood reduces availability for those who need it. Incorrect use is currently picked up by blood bank staff, there is no safety net in place.

Assessment of problem and analysis of its causes The list of red cells used for transfusion kept by bloodbank was audited, looking at who requested the transfusion and what special requirements they requested. This was compared against what each patient should have had requested, and discrepancies were identified.

Initially 2 months of blood requests were audited and showed $3 / 37$ and $7 / 57$ errors. There has therefore been discussion amongst haematology and blood bank across the city regarding measures to reduce this and re-audit conducted.

Intervention Three measures have been introduced:

- Alerts on the computer system for patients with special transfusion requirements

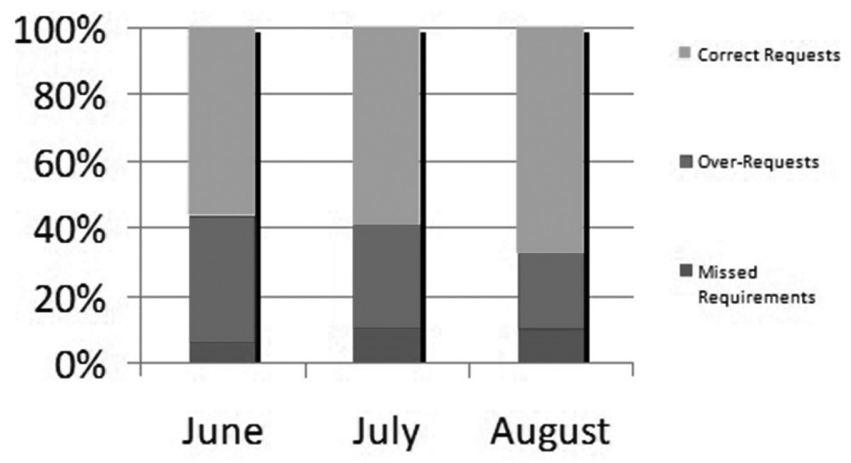

Abstract G548(P) Figure 1
- A citywide guideline of which scenarios or patients warrant these requirements

- A form held by blood bank for each patient detailing their requirements, created at their first transfusion

Study design This was an audit and had a prospective design Strategy for change The results were discussed in conjunction with other local haematology departments. The staff of the haematology/oncology unit were given access to the agreed guideline of which types of patients have special transfusion needs, trakcare alerts were implemented and blood bank agreed on forms that they would hold. These were in place for a year prior to reaudit. Following the results of re-audit, a presentation at a departmental meeting highlighted ongoing issues.

Measurement of improvement Following the interventions the results were analysed simply to show percentage change pre- and post intervention. The results showed a large increase in the number of incorrectly requested transfusions from an average of $10 \%$ error per month to $38 \%$ per month. The majority of errors were due to over-requesting special requirements (30\%), but there was also a significant amount of missed requirements $(8 \%)$. The majority of errors were related to CMV requesting (69\%).

The alerts on the computer system relating to special requirements were also looked at. $25 \%$ of patients had alerts. 6 of these had alerts when, according to the guideline, they are not required and 4 who do have special requirements did not have an alert.

Effects of changes There are still a high number of errors and this clearly is an ongoing problem with an impact on patient safety. On discussion several issues have been highlighted. Trakcare alerts have not been publicised to staff and there is limited knowledge of the intranet guideline, which is particularly relevant at night when the unit is covered by an on call team. Blood bank find the individual patient forms cumbersome and find it difficult to track down medical staff to fill them in.

Lessons learnt This audit has shown that there are ongoing errors in this area. The interventions in place have not fully addressed this and further work is needed.

Message for others This audit has identified a key patient safety issue. The misrequesting of blood can cause significant harm and the interventions tried so far have proved cumbersome. Further work is ongoing in this area.

\section{G549(P) LEARNING FROM EXCELLENCE: A NEW PARADIGM OF SAFETY REPORTING}

${ }^{1} \mathrm{~A}$ Plunkett, ${ }^{1} \mathrm{~N}$ Kelly, ${ }^{2} \mathrm{D}$ Scott. ${ }^{1}$ Paediatric Intensive Care, Birmingham Children's Hospital, Birmingham, UK; ' ${ }^{2}$ Governance Department, Birmingham Children's Hospital, Birmingham, UK

\subsection{6/archdischild-2015-308599.499}

Context This was a pilot study in a single department (Paediatric Intensive Care Unit) in a tertiary children's hospital.

Problem and Assessment of problem and analysis of its causes Safety in healthcare has traditionally focussed on reducing the rates of harm by learning from adverse events. This approach may miss opportunities to learn from episodes of clinical excellence. Furthermore, there is a potential negative impact on staff through the second victim phenomenon, whereby staff involved in adverse events may experience negative emotional consequences (e.g. fear and avoidance). 
Safety 2 is an emerging concept, advocating for learning from "what goes right", rather than "what goes wrong”. Currently, Safety 2 methodology is not well described in healthcare.

Intervention We piloted a system of peer-reporting for episodes of clinical excellence, to identify common themes of excellence practice. The "Learning from excellence" initiative was launched in April 2014 in our PICU. Reporting was accessible to all staff via a simple online form. Excellence was identified by individual reporters: a standardised definition of excellence was not provided.

Study design This is a descriptive study of themes identified from a system of peer-reported excellence in healthcare. All reports were analysed for themes in 2 domains:

1. Excellent practice (i.e. what was done?)

2. Clinical context.

Strategy for change The project was designed in conjunction with the Trust governance department. An online reporting form was published on the hospital intranet, and the initiative was championed by members of the PICU team. Reported individuals and teams were notified of their excellence citation via email. Briefings with summaries of excellence reports, highlighting learning opportunities are circulated to the department weekly. The next phase is to include the contents of these reports in the educational programme to develop role modelling and emulation of excellent practice. This process is underway at the time of writing.

Measurement of improvement To date, 74 reports have been submitted and analysed. Staff members from every clinical group have contributed to the reporting, and received reports. 19 practice themes and 24 discrete clinical context themes have been identified. Table 1 shows the 10 commonest themes in each domain.

Effects of changes There is a general trend of increasing numbers of reports since launch. The scope of the project is increasing through reporting outside the PICU. This has occurred without external championing, presumably via a "viral" spread.

At the time of writing we have not evaluated staff satisfaction or any impact on clinical metrics. However, the rising frequency of reporting, and the spread outside the unit provides evidence of proof of concept, and acceptance of potential utility.

Lessons learnt Involvement of the Trust governance department allowed us to use an existing infrastructure for reporting, and provided useful insights into safety management.

Championing within the environment of the intervention was essential.

\begin{tabular}{|c|c|c|c|}
\hline Practice theme & Occurrences & Clinical context theme & Occurrences \\
\hline Peer support & 17 & Prescribing & 7 \\
\hline Preparedness/planning & 12 & End of life & 6 \\
\hline Compassionate care & 5 & Admissions & 5 \\
\hline Timeliness & 5 & Resuscitation & 5 \\
\hline Clinical leadership & 4 & Sepsis & 5 \\
\hline Advocacy/courage & 4 & Complex discharges & 4 \\
\hline Communication with & & Arterial line & \\
\hline colleagues & 3 & management & 3 \\
\hline Communication with & & Expressed Breast & \\
\hline patients & 3 & Milk delivery & 2 \\
\hline Going the extra mile & 3 & Handover & 2 \\
\hline Teamwork & 3 & Transfer & 2 \\
\hline
\end{tabular}

Message for others We have demonstrated that peer-reported excellence can be captured from a wide range of themes and clinical contexts. We intend to use these reports to generate a positive movement to change, to inspire excellent practice through role modelling and emulation.

\section{G550(P) ASSESSING COMMUNICATION IN PHLEBOTOMY SERVICES IN AN IN-PATIENT SETTING}

B Singh, S Habeeb, J Pitchforth, C Lemer. General Paediatrics, Evalina Children's Hospital, London, UK

\subsection{6/archdischild-2015-308599.500}

Context Quality improvement project carried out on the general paediatrics ward involving the different medical teams, (general paediatrics, surgery, ENT, orthopaedics), the Nursing team and the phlebotomists

Problem We addressed the problem of the delay in the notification of failed blood tests from the phlebotomy team to the relevant medical team. This was identified to affect patient care by causing a delay in diagnosis, decisions about treatment and discharge, leading on to prolonging patient stay, this in affects the overall patient experience.

Assessment of problem and analysis of its causes The problem was quantified by assessing the total number of unsuccessful blood tests in 1 week and finding out how many of those had been communicated to the relevant medical team at the time. To understand how there could be possible breakdowns in communication, a day was spent with the phlebotomists to understand how they prioritise their jobs, how long they have per patient and what would be the best way for them to contact the medical team. The staff involved included junior doctors, phlebotomists and nurses.

Intervention At the end of each phlebotomy round, the phlebotomists bleep the nurse in charge for the ward and inform of any unsuccessful blood tests. The nurse in charge can then contact the relevant medical team so that they can act and decide whether the test is needed more urgently or can wait until the next phlebotomy round.

Strategy for change The change was implemented by being discussed at the weekly grand round session so that all staff were aware. Staff involved in the change included the medical team, nursing team and phlebotomists. The results were presented at an audit meeting and a re-audit was performed 1 year later showing a $100 \%$ success rate and that change had been sustained. These were results were then represented at junior

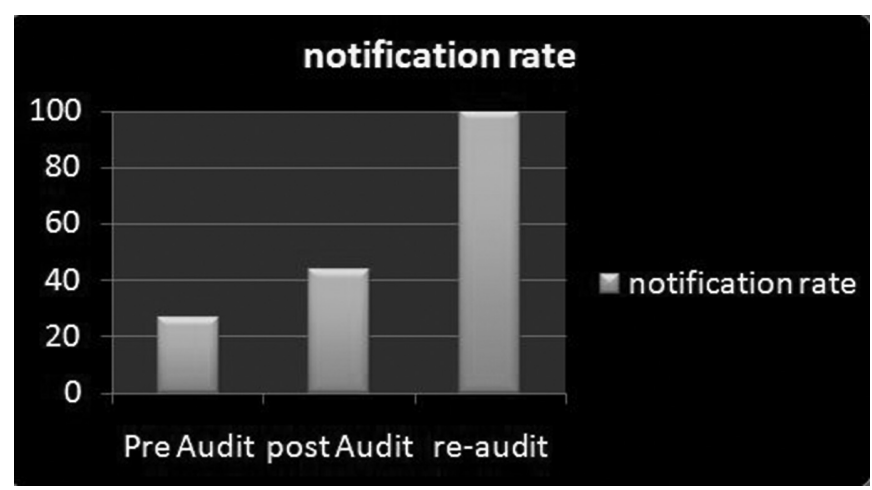

Abstract G550(P) Figure 1 